\title{
Predictors of acute kidney injury after paraquat intoxication
}

\author{
Cheng-Hao Weng ${ }^{1, *}$, Hui-Hsiang Chen ${ }^{1, *}$, Ching-Chih $\mathrm{Hu}^{2}$, Wen-Hung Huang ${ }^{1}$, \\ Ching-Wei Hsu ${ }^{1}$, Jen-Fen Fư ${ }^{3}$, Wey-Ran Lin ${ }^{4}$, I-Kwan Wang ${ }^{5}$ and Tzung-Hai Yen ${ }^{1,6,7}$ \\ ${ }^{1}$ Department of Nephrology and Poison Center, Chang Gung Memorial Hospital and College of Medicine, Chang Gung \\ University, Linkou, Taiwan \\ ${ }^{2}$ Department of Hepatogastroenterology and Liver Research Unit, Chang Gung Memorial Hospital, Keelung, Taiwan \\ ${ }^{3}$ Department of Medical Research, Chang Gung Memorial Hospital and College of Medicine, Chang Gung University, Linkou, \\ Taiwan \\ ${ }^{4}$ Department of Gastroenterology and Hepatology, Chang Gung Memorial Hospital and College of Medicine, Chang Gung \\ University, Linkou, Taiwan \\ ${ }^{5}$ Department of Nephrology, Chang Medical University Hospital and College of Medicine, China Medical University, Taichung, \\ Taiwan \\ ${ }^{6}$ Kidney Research Center, Chang Gung Memorial Hospital, Linkou, Taiwan \\ ${ }^{7}$ Center for Tissue Engineering, Chang Gung Memorial Hospital, Linkou, Taiwan \\ *These authors contributed equally to this work
}

Correspondence to: Tzung-Hai Yen, email: m19570@adm.cgmh.org.tw

Keywords: paraquat, suicide, acute kidney injury, SOFA, AKIN

Received: March 14, 2017 Accepted: May 07, $2017 \quad$ Published: May 18, 2017

Copyright: Weng et al. This is an open-access article distributed under the terms of the Creative Commons Attribution License 3.0 (CC BY 3.0), which permits unrestricted use, distribution, and reproduction in any medium, provided the original author and source are credited.

\section{ABSTRACT}

Paraquat intoxication is characterized by multi-organ failure, causing substantial mortality and morbidity. Many paraquat patients experience acute kidney injury (AKI), sometimes requiring hemodialysis. We observed 222 paraquat-intoxicated patients between 2000 and 2012, and divided them into AKI $(n=103)$ and nonAKI $(n=119)$ groups. The mortality rate was higher for AKI than non-AKI patients (70.1\% vs. $40.0 \%, P<0.001)$. Patients with AKI had a longer time to hospital arrival $(P=0.003)$, lower $\mathrm{PaO}_{2}(P=0.006)$ and higher alveolar-arterial $\mathrm{O}_{2}$ difference $(P<0.001) 48 \mathrm{~h}$ after admission, higher sequential organ failure assessment 48 -h score $(P<0.001)$, higher severity index of paraquat poisoning (SIPP) score $(P=0.016)$, lower $\mathrm{PaCO}_{2}$ at admission $(P=0.031)$, higher $\mathrm{PaO}_{2}$ at admission $(P=0.015)$, lower nadir $\mathrm{PaCO}_{2}(P=0.001)$ and lower nadir $\mathrm{HCO}_{3}(P=0.004)$ than non-AKI patients. Multivariate logistic regression indicated that acute hepatitis $(P<0.001)$, a longer time to hospital arrival $(P<0.001)$, higher SIPP score $(P=0.026)$ and higher $\mathrm{PaO}_{2}$ at admission $(P=0.014)$ were predictors of AKI. The area under the receiver operating characteristic curve confirmed that an Acute Kidney Injury Network 48-hour score $\geq 2$ predicted AKI necessitating hemodialysis with a sensitivity of 0.6 and specificity of 0.832 . AKI is common (46.4\%) following paraquat ingestion, and acute hepatitis, the time to hospital arrival, SIPP score and $\mathrm{PaO}_{2}$ at admission were powerful predictors of AKI. Larger studies with longer follow-up durations are warranted.

\section{INTRODUCTION}

Paraquat is a contact herbicide with extremely high toxicity. Paraquat is easily accessible, and in Taiwan, it is commonly ingested, whether deliberately or unintentionally [1]. Due to its severe toxicity, paraquat consumption is fatal in $60-80 \%$ of cases. Patients who have consumed about $40 \mathrm{~mL}$ of a $24 \%$ paraquat solution typically die of multiple organ failure in the next few hours or days [2]. Upon ingestion, paraquat is absorbed quickly, although not completely, and is mostly excreted in the urine without further metabolism within 12-24 
hours. Although the characteristic clinical features of paraquat intoxication are acute lung injury and multiple organ failure, acute kidney injury (AKI) also frequently occurs after acute large-dose exposure to this toxic herbicide [3].

Any patient who comes to our hospital with paraquat intoxication is treated with a standard detoxification protocol including charcoal hemoperfusion, pulse therapies with methylprednisolone and cyclophosphamide, and extended treatment with dexamethasone $[4,5]$. For most patients, this practice nearly normalizes respiratory function and blood oxygen concentrations within three to six months [6]. This protocol has been reviewed and recommended by the Cochrane Injuries Group as beneficial in cases of lung fibrosis caused by paraquat [7]. Many paraquat patients experience AKI, and some may still progress to AKI necessitating hemodialysis (HD) despite intensive treatment.

The mechanism whereby paraquat causes AKI is not fully understood; however, it is known that this compound can accrue within renal tubular cells, leading to cycles of reduction and oxidation, generating reactive oxygen species, and ultimately damaging the proximal tubules [8]. As the kidney is the main organ responsible for paraquat excretion, the resultant kidney injury may reduce the elimination of paraquat and increase its toxicity in other organs [9]. Mohamed et al. [10] demonstrated that kidney damage biomarkers such as neutrophil gelatinase-associated lipocalin were upregulated in AKI, but did not independently predict death. Only functional markers such as serum creatinine predicted mortality after paraquat intoxication. Reduced kidney function is only one factor causing the rapid rise in creatinine after paraquat poisoning [11]. During severe oxidative stress, it is likely that higher levels of creatine and creatinine are generated to meet the increased need for energy. To a lesser degree, acidosis may increase creatine cyclisation to creatinine, or creatinine secretion may be competitively or noncompetitively inhibited.

Kim et al. [12] reported that hyperuricemia increased the risk of mortality 3.7-fold and increased the risk of kidney failure 3.3-fold (adjusting for age, sex and estimated paraquat intake), and proposed that the serum level of uric acid was a reliable clinical predictor of death or AKI in patients with acute paraquat poisoning. In our previous studies $[13,14]$, we did not identify a strong correlation between the serum uric acid level and mortality. Theoretically, kidney failure reduces the elimination of uric acid and might increase serum uric acid levels.

In this study, we investigated the clinical features, AKI spectrum and outcomes in patients with deliberate paraquat intoxication, and most importantly, we evaluated the predictors of AKI after paraquat intoxication.

\section{RESULTS}

\section{Subject characteristics}

As shown in Table 1, the mortality rate was higher for AKI patients than for non-AKI patients $(70.1 \%$ vs. $40.0 \%$, $P<0.001)$. Furthermore, AKI patients exhibited a longer time to hospital arrival $(17.7 \pm 24.5$ vs. $9.9 \pm 17.1$ hours, $P=0.003$ ), lower $\mathrm{PaO}_{2} 48$ hours after admission $(56.0 \pm 26.0$ vs. $65.9 \pm 23.0 \mathrm{mmHg}, P=0.006)$, higher alveolar-arterial oxygen gradient $\left(\mathrm{AaDO}_{2}\right) 48$ hours after admission $(56.1 \pm 27.9$ vs. $38.4 \pm 26.2 \mathrm{mmHg}$, $P<0.001)$, higher $\mathrm{SOFA}_{48 \mathrm{~h}}$ score $(4.7 \pm 2.1$ vs. $1.8 \pm 1.5$, $P<0.001)$, higher SIPP score $(0.7 \pm 0.5$ vs. $0.5 \pm 0.5$, $P=0.016)$, lower partial pressure of arterial carbon dioxide $\left(\mathrm{PaCO}_{2}\right)$ at admission $(31.0 \pm 10.5$ vs. $34.2 \pm 8.1$ mmHg, $P=0.031)$, higher $\mathrm{PaO}_{2}$ at admission $(88.5 \pm 20.4$ vs. $81.8 \pm 17.1 \mathrm{mmHg}, P=0.015$ ), lower nadir $\mathrm{PaCO}_{2}$ $(30.1 \pm 9.0$ vs. $36.8 \pm 14.0 \mathrm{mmHg}, P=0.001)$ and lower nadir bicarbonate $\left(\mathrm{HCO}_{3}\right)(18.3 \pm 7.3$ vs. $21.3 \pm 6.0$ $\mathrm{mmHg}, P=0.004)$ than non-AKI patients.

In addition, the mortality rate tended to be higher for AKI HD patients than for non-AKI HD patients, although this trend was not statistically significant $(80.0 \%$ vs. $50.9 \%, P=0.107$, Table 2 ). Nevertheless, the AKI HD group demonstrated a higher risk of ARDS (50.0\% vs. $25.1 \%, P=0.032$ ), higher $\mathrm{AaDO}_{2} 48$ hours after admission $(58.5 \pm 31.9$ vs. $45.2 \pm 27.7 \mathrm{mmHg}, P=0.047)$, higher SOFA $_{48 \mathrm{~h}}$ score $(4.5 \pm 2.5$ vs. $3.0 \pm 2.2, P=0.007)$, higher SIPP score $(0.9 \pm 0.4$ vs. $0.5 \pm 0.5, P=0.001)$, and lower $\mathrm{PaCO}_{2}$ at admission ( $28.2 \pm 8.1$ vs. $34.3 \pm 12.6$ $\mathrm{mmHg}, P=0.034$ ) than the non-AKI HD group.

\section{Predictors of AKI}

Multivariate logistic regression analysis demonstrated that acute hepatitis $(P<0.001)$, a longer time to hospital arrival $(P<0.001)$, higher SIPP score $(P=0.026)$ and higher $\mathrm{PaO}_{2}$ at admission $(P=0.014)$ were significant predictors of AKI (Table 3). The sensitivity, specificity and cutoff values of these predictors of AKI are summarized in Table 5.

\section{Predictor of AKI HD}

Multivariate logistic regression analysis indicated that a higher $\mathrm{AKIN}_{48 \mathrm{~h}}$ score $(P<0.001)$ was a significant predictor of AKI HD (Table 4). The sensitivity, specificity and cutoff value of this predictor of AKI HD are summarized in Table 5. AUROC analysis revealed that an $\mathrm{AKIN}_{48 \mathrm{~h}}$ score $=1.5$ was the cutoff point for the prediction of AKI HD. Finally, an AKIN $_{48 h}$ score $\geq 2$ predicted AKI HD with a sensitivity of 0.6 and specificity of 0.832 (Figure 1). 
Table 1: Comparisons between paraquat patients with and without AKI $(n=222)$

\begin{tabular}{|c|c|c|c|}
\hline & AKI $(n=103)$ & Non-AKI $(n=119)$ & $P$ \\
\hline Age & $41.8 \pm 15.9$ & $42.3 \pm 15.1$ & 0.825 \\
\hline Gender (male), $n(\%)$ & $86(83.9 \%)$ & $86(72.0 \%)$ & 0.056 \\
\hline Mortality, $n(\%)$ & $72(70.1 \%)$ & $48(40.0 \%)$ & $<0.001 * * *$ \\
\hline ARDS, $n(\%)$ & $33(32.2 \%)$ & $29(24.0 \%)$ & 0.253 \\
\hline Acute lung injury, $n(\%)$ & $64(62.1 \%)$ & $48(40.0 \%)$ & 0.479 \\
\hline Pneumomediastinum, $n(\%)$ & $8(7.8 \%)$ & 0 & $0.002 * *$ \\
\hline Time to hospital arrival (hours) & $17.7 \pm 24.5$ & $9.9 \pm 17.1$ & $0.003^{* *}$ \\
\hline Estimated ingestion amount (mL) & $80.8 \pm 77.3$ & $81.0 \pm 124.1$ & 0.987 \\
\hline Blood paraquat concentration (ppm) & $4.6 \pm 4.6$ & $4.9 \pm 6.4$ & 0.731 \\
\hline Peak ALT concentration (U/L) & $190.9 \pm 161.3$ & $176.5 \pm 69.5$ & 0.442 \\
\hline Peak AST concentration (U/L) & $122.4 \pm 82.8$ & $132.9 \pm 247.8$ & 0.909 \\
\hline Peak bilirubin concentration $(\mathrm{mg} / \mathrm{dL})$ & $3.31 \pm 2.1$ & $3.0 \pm 1.2$ & 0.279 \\
\hline Nadir $\mathrm{PaO}_{2}(\mathrm{mmHg})$ & $56.0 \pm 26.0$ & $65.9 \pm 23.0$ & $0.006^{* *}$ \\
\hline Nadir $\mathrm{AaDO}_{2}(\mathrm{mmHg})$ & $56.1 \pm 27.9$ & $38.4 \pm 26.2$ & $<0.001 * * *$ \\
\hline $\mathrm{SOFA}_{48 \mathrm{~h}}$ & $4.7 \pm 2.1$ & $1.8 \pm 1.5$ & $<0.001 * * *$ \\
\hline SIPP score (hours/mg/L) & $0.7 \pm 0.5$ & $0.5 \pm 0.5$ & $0.016^{*}$ \\
\hline $\mathrm{PaCO}_{2}$ at admission $(\mathrm{mmHg})$ & $31.0 \pm 10.5$ & $34.2 \pm 8.1$ & $0.031 *$ \\
\hline $\mathrm{PaO}_{2}$ at admission $(\mathrm{mmHg})$ & $88.5 \pm 20.4$ & $81.8 \pm 17.1$ & $0.015^{*}$ \\
\hline Nadir $\mathrm{PaCO}_{2}(\mathrm{mmHg})$ & $30.1 \pm 9.0$ & $36.8 \pm 14.0$ & $<0.001 * * *$ \\
\hline Nadir $\mathrm{HCO}_{3}(\mathrm{mmHg})$ & $18.3 \pm 7.3$ & $21.3 \pm 6.0$ & $0.004 * *$ \\
\hline
\end{tabular}

Note: $* P<0.05, * * P<0.01, * * * P<0.001$, AKI acute kidney injury, ARDS acute respiratory distress syndrome, $\mathrm{AaDO}_{2}$ alveolar-arterial oxygen gradient, ALT alanine aminotransferase, AST aspartate aminotransferase, $\mathrm{HCO}_{3}$ bicarbonate, $\mathrm{PaO}_{2}$ partial pressure of arterial oxygen, $\mathrm{PaCO}_{2}$ partial pressure of arterial carbon dioxide, SOFA sequential organ failure assessment, SIPP severity index of paraquat poisoning

\section{DISCUSSION}

The present cohort data are important because a large sample was included $(n=222)$, and a standard detoxification protocol was used to treat all paraquat-intoxicated patients: charcoal hemoperfusion, methylprednisolone and cyclophosphamide pulse therapies, and extensive treatment with dexamethasone. As shown in Table 6, the published mortality rates and AKI incidence rates after paraquat poisoning range from $24.0-73.9 \%$ and $50.7-100.0 \%$, respectively $[2,3,10-12,15-18]$. The overall mortality rate in this study was $54.1 \%$, and AKI was common (46.4\%) following paraquat ingestion. Some clinically useful variables such as acute hepatitis, the time to hospital arrival, SIPP score and $\mathrm{PaO}_{2}$ at admission were powerful predictors of AKI. Finally, an $\mathrm{AKIN}_{48 \mathrm{~h}}$ score $\geq 2$ predicted AKI HD with a sensitivity of 0.6 and specificity of 0.832 .

Acute hepatitis was a significant predictor of AKI after paraquat intoxication. The mechanisms of paraquat toxicity involve the generation of superoxide anions and the subsequent generation of more harmful reactive oxygen species such as hydrogen peroxide and hydroxyl radical. In addition, nicotinamide adenine dinucleotide phosphate hydrogen (NADPH) is oxidized as it supplies the majority of reducing equivalents to reduce paraquat intracellularly, which thus impairs critical NADPHrequiring biochemical processes [19]. Liver damage has been reported in numerous studies. DeGray et al. [20] proposed that NADPH-cytochrome P-450 reductase reduced paraquat to its radical cations in rat hepatocytes. An additional study [21] evaluated oxidative liver injury 20 hours after oral paraquat intake (a single dose of $150 \mathrm{mg} / \mathrm{kg}$ ) in rats. Paraquat did not alter the activity of cytosolic superoxide dismutase, but enhanced the activity of mitochondrial superoxide dismutase by $14.0 \%$. Glutathione levels were reduced by $30.0 \%$, and the ratio of glutathione to glutathione disulfide was reduced by $50 \%$. Since mitochondrial superoxide dismutase activity increased while glutathione levels decreased following paraquat intake, the hepatic toxicity of paraquat appeared to be due to the superoxide anion [21].

Since paraquat is eliminated by the kidneys, the ultimate result of paraquat poisoning largely depends on the extent of kidney damage. The nephrotoxicity of paraquat is thought to arise from cycles of reduction and oxidation and the generation of reactive oxygen species [8]. 
Table 2: Comparisons between paraquat patients with and without AKI HD $(n=222)$

\begin{tabular}{|c|c|c|c|}
\hline & AKI HD $(n=24)$ & Non-AKI HD $(n=198)$ & $\boldsymbol{P}$ \\
\hline Age & $38.0 \pm 10.9$ & $42.6 \pm 15.8$ & 0.204 \\
\hline Gender (male), $n(\%)$ & $17(70 \%)$ & $155(78.4 \%)$ & 0.401 \\
\hline Mortality, $n(\%)$ & $19(80 \%)$ & $101(50.9 \%)$ & 0.107 \\
\hline ARDS, $n(\%)$ & $12(50.0 \%)$ & $50(25.1 \%)$ & $0.032 *$ \\
\hline Acute lung injury, $n(\%)$ & $14(60.0 \%)$ & $98(49.4 \%)$ & 0.479 \\
\hline Pneumomediastinum, $n(\%)$ & $3(12.5 \%)$ & $5(2.5 \%)$ & $0.047 *$ \\
\hline Time to hospital arrival (hours) & $15.0 \pm 19.7$ & $13.3 \pm 21.3$ & 0.744 \\
\hline Estimated ingestion amount (mL) & $77.1 \pm 82.8$ & $81.4 \pm 107.2$ & 0.862 \\
\hline Blood paraquat concentration (ppm) & $4.8 \pm 3.5$ & $4.8 \pm 5.8$ & 0.992 \\
\hline Peak ALT concentration (U/L) & $239.5 \pm 220.5$ & $176.5 \pm 102.1$ & 0.221 \\
\hline Peak AST concentration (U/L) & $189.5 \pm 174.7$ & $120.6 \pm 139.1$ & 0.508 \\
\hline Peak bilirubin concentration (mg/dL) & $3.1 \pm 0.7$ & $3.2 \pm 1.8$ & 0.868 \\
\hline Nadir $\mathrm{PaO}_{2}(\mathrm{mmHg})$ & $56.2 \pm 34.1$ & $61.9 \pm 23.6$ & 0.477 \\
\hline Nadir $\mathrm{AaDO}_{2}(\mathrm{mmHg})$ & $58.5 \pm 31.9$ & $45.2 \pm 27.7$ & $0.047 *$ \\
\hline $\mathrm{SOFA}_{48 \mathrm{~h}}$ & $4.5 \pm 2.5$ & $3.0 \pm 2.2$ & $0.007 * *$ \\
\hline SIPP score (hours/mg/L) & $0.9 \pm 0.4$ & $0.5 \pm 0.5$ & $0.001 * *$ \\
\hline $\mathrm{PaCO}_{2}$ at admission $(\mathrm{mmHg})$ & $29.3 \pm 8.5$ & $33.0 \pm 9.5$ & 0.169 \\
\hline $\mathrm{PaO}_{2}$ at admission $(\mathrm{mmHg})$ & $91.1 \pm 20.5$ & $84.2 \pm 18.7$ & 0.123 \\
\hline Nadir $\mathrm{PaCO}_{2}(\mathrm{mmHg})$ & $28.2 \pm 8.1$ & $34.3 \pm 12.6$ & $0.034 *$ \\
\hline Nadir $\mathrm{HCO}_{3}(\mathrm{mmHg})$ & $18.0 \pm 6.6$ & $20.1 \pm 6.8$ & 0.180 \\
\hline
\end{tabular}

Note: $* P<0.05,{ }^{*} P<0.01$, AKI HD acute kidney injury necessitating hemodialysis, ARDS acute respiratory distress syndrome, $\mathrm{AaDO}_{2}$ alveolar-arterial oxygen gradient, ALT alanine aminotransferase, AST aspartate aminotransferase, $\mathrm{HCO}_{3}$ bicarbonate, $\mathrm{PaO}_{2}$ partial pressure of arterial oxygen, $\mathrm{PaCO}_{2}$ partial pressure of arterial carbon dioxide, SOFA sequential organ failure assessment, SIPP severity index of paraquat poisoning.

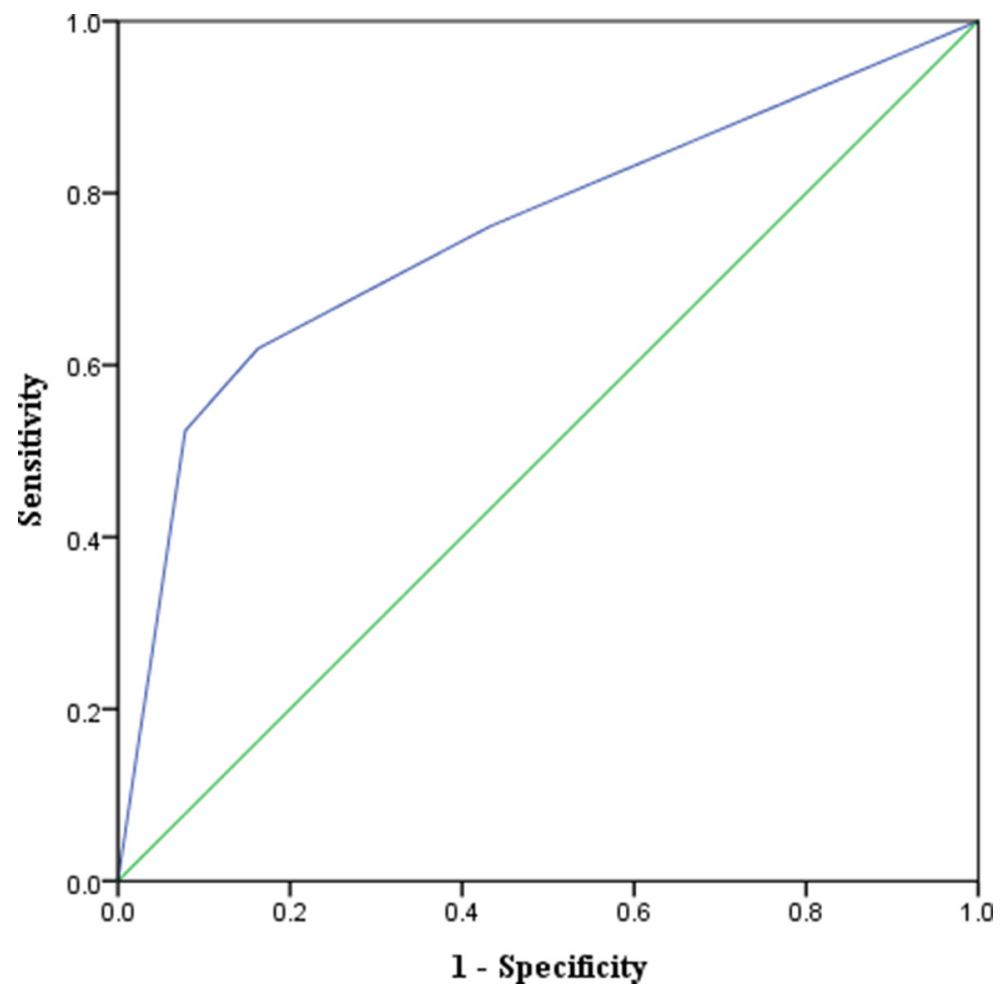

Figure 1: Area under the receiver operating characteristic curve (AUROC) analysis. It was revealed that an $\mathrm{AKIN}_{48 \mathrm{~h}} \mathrm{score} \geq 2$ had an AUROC of $0.741 \pm 0.068$ and predicted AKI HD with a sensitivity of 0.6 and specificity of 0.832 . 
Table 3: Analysis of AKI with univariate and multivariate logistic regression models $(n=222)$

\begin{tabular}{|c|c|c|c|c|}
\hline & B & SE & $\operatorname{Exp}(B)$ & $\boldsymbol{P}$ \\
\hline \multicolumn{5}{|l|}{ Univariate } \\
\hline Time to hospital arrival (hours) & 0.019 & 0.008 & $1.019(1.004-1.035)$ & $0.015^{*}$ \\
\hline SIPP score (hours/mg/L) & 0.722 & 0.302 & $2.058(1.140-3.717)$ & $0.017 *$ \\
\hline Acute hepatitis & 1.740 & 0.322 & $5.697(3.033-10.699)$ & $<0.001 * * *$ \\
\hline $\mathrm{PaO}_{2}$ at admission $(\mathrm{mmHg})$ & 0.020 & 0.008 & $1.020(1.003-1.037)$ & $0.018^{*}$ \\
\hline Nadir $\mathrm{PaO}_{2}(\mathrm{mmHg})$ & -0.017 & 0.006 & $0.983(0.971-0.996)$ & $0.008 * *$ \\
\hline Nadir $\mathrm{PaCO}_{2}(\mathrm{mmHg})$ & -0.066 & 0.018 & $0.936(0.904-0.969)$ & $<0.001 * * *$ \\
\hline Nadir $\mathrm{HCO}_{3}(\mathrm{mmHg})$ & -0.065 & 0.023 & $0.937(0.896-0.979)$ & $0.004 * *$ \\
\hline Nadir $\mathrm{AaDO}_{2}(\mathrm{mmHg})$ & 0.024 & 0.006 & $1.025(1.013-1.037)$ & $<0.001 * * *$ \\
\hline Acute lung injury & 0.881 & 0.301 & $2.414(1.337-4.356)$ & $0.003 * *$ \\
\hline \multicolumn{5}{|l|}{ Multivariate } \\
\hline Acute hepatitis & 2.230 & 0.414 & $9.301(4.835-25.462)$ & $<0.001 * * *$ \\
\hline Time to hospital arrival (hours) & 0.045 & 0.010 & $1.046(1.030-1.074)$ & $<0.001 * * *$ \\
\hline SIPP score (hours/mg/L) & 0.958 & 0.429 & $2.606(1.066-6.010)$ & $0.026^{*}$ \\
\hline $\mathrm{PaO}_{2}$ at admission $(\mathrm{mmHg})$ & 0.027 & 0.011 & $1.027(1.008-1.053)$ & $0.014 *$ \\
\hline
\end{tabular}

Note: $* P<0.05,{ }^{*} P<0.01$, B beta coefficient or regression coefficient, Exp (B) exponential B or odds ratio, AKI acute kidney injury, ARDS acute respiratory distress syndrome, $\mathrm{AaDO}_{2}$ alveolar-arterial oxygen gradient, ALT alanine aminotransferase, AST aspartate aminotransferase, $\mathrm{HCO}_{3}$ bicarbonate, $\mathrm{PaO}_{2}$ partial pressure of arterial oxygen, $\mathrm{PaCO}_{2}$ partial pressure of arterial carbon dioxide, SE standard error, SOFA sequential organ failure assessment, SIPP severity index of paraquat poisoning.

Table 4: Analysis of AKI HD with univariate and multivariate logistic regression models $(n=222)$

\begin{tabular}{lcccc}
\hline & B & SE & Exp (B) & $P$ \\
\hline Univariate & & & & \\
Pneumomediastinum & 1.744 & 0.774 & $5.718(1.255-26.040)$ & $0.024^{*}$ \\
ARDS & 1.320 & 0.495 & $3.743(1.418-9.879)$ & $<0.001^{* * *}$ \\
Creatinine at admission (mg/dL) & 0.312 & 0.105 & $1.366(1.113-1.667)$ & $0.003^{* *}$ \\
AKIN $_{48 \mathrm{~h}}$ & 0.895 & 0.213 & $2.448(1.613-3.716)$ & $<0.001^{* * *}$ \\
Nadir AaDO $_{2}(\mathrm{mmHg})$ & 0.017 & 0.009 & $1.017(1.000-1.035)$ & 0.05 \\
SOFA $_{48 \mathrm{~h}}$ & 0.241 & 0.094 & $1.273(1.059-1.530)$ & $0.010^{*}$ \\
Multivariate $_{\text {AKIN }}$ & & & & $<0.001^{* * *}$ \\
\hline
\end{tabular}

Note: $* P<0.05, * * P<0.01$, AKI HD acute kidney injury necessitating hemodialysis, AKIN Acute Kidney Injury Network, ARDS acute respiratory distress syndrome, $\mathrm{AaDO}_{2}$ alveolar-arterial oxygen gradient, $\mathrm{B}$ beta coefficient or regression coefficient, Exp (B) exponential B or odds ratio, SE standard error, SOFA sequential organ failure assessment

The association between AKI and liver cirrhosis is a well-recognized disease entity [22]. Acute liver failure is frequently accompanied by concurrent AKI as a result of renal hypoperfusion, the nephrotoxicity of the drug itself, or sepsis and systemic inflammation, which causes further morbidity and correlates greatly with a poor prognosis [23]. Nevertheless, our earlier study [2] indicated that while many paraquat patients experienced hepatic complications $(46.5 \%)$, their toxic hepatitis appeared to be mild and short-lived.
A greater time to hospital arrival and SIPP score were found to predict AKI after paraquat intoxication. This suggested that both the blood paraquat concentration and the time between paraquat ingestion and hospital arrival were significant determinants of AKI. The SIPP score has traditionally been used as a prognostic indicator following paraquat poisoning; a $\operatorname{SIP} P$ value $>10$ is usually associated with mortality [24-26]. We also found that SIPP scores differed significantly between AKI and non-AKI patients $(0.7 \pm 0.5$ vs. $0.5 \pm 0.5, P=0.016)$ and 
Table 5: Sensitivities, specificities and cutoff values of the predictors of AKI and AKI HD $(n=222)$

\begin{tabular}{lcccccc}
\hline & Sensitivity & Specificity & $\begin{array}{c}\text { Cutoff } \\
\text { value }\end{array}$ & AUROC \pm SE & 95\% CI & $P$ \\
\hline AKI & & & & & & \\
Time to hospital arrival (hours) & 0.483 & 0.740 & 6.5 & $0.625 \pm 0.041$ & $0.544-0.705$ & $0.003^{* *}$ \\
SIPP score (hours/mg/L) & 0.817 & 0.432 & 1.857 & $0.647 \pm 0.043$ & $0.562-0.731$ & $0.001^{* *}$ \\
$\mathrm{PaO}_{2}$ at admission (mmHg) & 0.816 & 0.310 & 76.7 & $0.580 \pm 0.042$ & $0.498-0.662$ & $0.049^{*}$ \\
$\mathrm{AKI} \mathrm{HD}$ & 0.6 & 0.832 & 2 & $0.741 \pm 0.068$ & $0.609-0.874$ & $<0.001^{* * *}$ \\
$\mathrm{AKIN}_{48 \mathrm{~h}}$ & & & & & & \\
\hline
\end{tabular}

Note: $* P<0.05, * * P<0.01, * * * P<0.001$, AUROC area under receiver operating characteristic curve, CI confidence interval, SE standard error, AKIN Acute Kidney Injury Network, AKI acute kidney injury, AKI HD AKI necessitating hemodialysis, SIPP severity index of paraquat poisoning.

\begin{tabular}{|c|c|c|c|c|c|}
\hline Study & Year & Area & Sample size & Mortality rate (\%) & AKI incidence rate $(\%)$ \\
\hline Current study & 2017 & Taiwan & 222 & 54.1 & 46.4 \\
\hline Mohamed et al. [10] & 2015 & Sri Lanka & 50 & 24.0 & 76.0 \\
\hline Mohamed et al. [11] & 2015 & Sri Lanka & 66 & 25.8 & 56.0 \\
\hline Yang et al. [2] & 2012 & Taiwan & 187 & 54.5 & 65.2 \\
\hline Seok et al. [15] & 2012 & Korea & 41 & 51.2 & 100.0 \\
\hline Moon et al. [16] & 2011 & Korea & 134 & 73.9 & 50.7 \\
\hline Kim et al. [12] & 2011 & Korea & 247 & 42.9 & 64.0 \\
\hline Gil et al. [17] & 2009 & Korea & 20 & 35.0 & 55.0 \\
\hline Kim et al. [3] & 2009 & Korea & 278 & 58.8 & 51.4 \\
\hline Koo et al. [18] & 2002 & Korea & 80 & 65.0 & 58.8 \\
\hline
\end{tabular}

Note: AKI acute kidney injury.

between AKI HD and non-AKI HD patients $(0.9 \pm 0.4$ vs. $0.5 \pm 0.5, P=0.001)$. Additionally, our previous study [5] confirmed that early charcoal hemoperfusion $<4$ hours $(P=0.020)$ or charcoal hemoperfusion $<5$ hours $(P=0.019)$ significantly reduced the risk of death in severely paraquat-poisoned patients. Similarly, Kim et al. [3] reported that paraquat intoxication was characterized by full-blown AKI after five days, but a return to normal kidney function within three weeks. Furthermore, the risk of mortality in the AKI group was significantly greater than that in the non-AKI group.

A higher $\mathrm{PaO}_{2}$ at admission was a significant predictor of AKI after paraquat intoxication. Previously [6], our group found that the respiratory functions and arterial blood gas levels of individuals who had survived paraquat poisoning were slowly restored to more appropriate levels after three months, even when lung fibrosis had occurred. Thus, it appears that severe lung inflammation (rather than pulmonary fibrosis) is the main contributor to lethal hypoxemia when paraquat poisoning is still subacute. Methylprednisolone pulse therapy and dexamethasone are powerful reducers of inflammation. Following dexamethasone therapy, pulse therapy can reduce the profound inflammation resulting from paraquat intoxication; as we previously reported, this enhanced the survival of patients with paraquat intoxication [27-29]. Furthermore, pulse therapy with methylprednisolone can prevent the release of certain factors from lymphocytes which would otherwise stimulate the generation of superoxide anions by macrophages and neutrophils [30]. Steroid treatment can also reduce superoxide production in the arachidonic acid cascade. For these reasons, recurrent pulse therapy with methylprednisolone can stop superoxide from further damaging the lungs and subsequently causing inflammation.

A higher $\mathrm{AKIN}_{48 \mathrm{~h}}$ score significantly predicted AKI HD after paraquat intoxication. In our previous study [14], the patient's age, time until hospitalization, circulating level of paraquat, estimated glomerular filtration rate at admission, and SOFA $_{48 \mathrm{~h}}$ score, but not $\mathrm{AKIN}_{48 \mathrm{~h}}$ score, significantly predicted death after paraquat intoxication. Nevertheless, Balestracci et al. [31] demonstrated that 
AKIN stage 3 at admission predicted AKI HD in patients with hemolytic uremic syndrome with a sensitivity of $92 \%$ and specificity of $84.2 \%$. Furthermore, Kielstein et al. [32] reported that the majority of patients undergoing interventional lung assist treatment and receiving extended hemodialysis were in AKIN stage 3. Although most studies have reported that an $\mathrm{AKIN}_{48 \mathrm{~h}}$ score $\geq 3$ predicts mortality [33-35], we found that an $\mathrm{AKIN}_{48 \mathrm{~h}}$ score $\geq 2$ predicted AKI HD. There was no clear explanation for this, though it may have been due to severe multi-organ damage following large-dose exposure to paraquat.

ARDS was a significant predictor of AKI HD in univariate analysis, but not after multivariate analysis. ARDS was independently associated with AKI in critically ill patients [36]. A high percentage $(34.0 \%)$ of patients undergoing interventional lung assist treatment received extended hemodialysis for AKI [32]. When ARDS concurs with AKI, the duration of stay in the intensive care unit and use of resources both increase, and the mortality rate markedly increases to nearly $60 \%$ [37]. Recent studies have demonstrated that ARDS can cause AKI, and AKI can alter leukocyte trafficking and pulmonary vascular permeability [37]. The lungs and kidneys receive a high circulating volume of blood every minute. The lungs are exposed to the entire cardiac output, and the kidneys are exposed to $22.0 \%$ of the cardiac output. Therefore, during paraquat intoxication, both organs are exposed to large amounts of paraquat and the accompanying circulating inflammatory mediators, neutrophils and monocytes, and are thus at risk for injury [38].

Similarly, pneumomediastinum was a significant predictor of AKI HD in univariate analysis, but not after multivariate analysis. This may be because too few paraquat patients (only 8/222) suffered from pneumomediastinum to reveal a significant impact on AKI HD. The Macklin effect may explain why paraquat-intoxicated patients develop pneumomediastinum [39]. Paraquat poisoning results in stiff lung and a predisposition to barotrauma. After the alveoli have ruptured, free air may travel toward the hilum of the lung via peribronchial vascular sheaths, and then spread proximally to the mediastinum. Zhou et al. [40] reported that pneumomediastinum occurred in $21.3 \%$ of their paraquat-intoxicated patients (16/75). Thirteen of these patients experienced pneumomediastinum within three days of consuming paraquat, and 15 died within three days of the onset of pneumomediastinum. Toxic hepatitis $(P=0.008)$, respiratory insufficiency $(P=0.003)$ and acute renal failure $(P=0.001)$ occurred more frequently in patients with pneumomediastinum than in those without. Finally, pneumomediastinum was found to predict a greater risk of 90 -day $(P=0.045)$ and 5-day mortality $(P=0.017)[40]$.

In summary, AKI was common (46.4\%) following paraquat ingestion, and certain clinically useful variables such as acute hepatitis, the time to hospital arrival, SIPP score and $\mathrm{PaO}_{2}$ at admission were powerful predictors of AKI. A higher AKIN ${ }_{48 \mathrm{~h}}$ score significantly predicted AKI HD after paraquat intoxication. Nevertheless, as this study was retrospective, included a small population of patients and involved a short period of follow-up, further studies are warranted to confirm our results.

\section{MATERIALS AND METHODS}

\section{Ethics statement}

This retrospective observational study complied with the guidelines of the Declaration of Helsinki and was approved by the Medical Ethics Committee of Chang Gung Memorial Hospital. Since this study involved the retrospective review of existing data, approval from the Institutional Review Board was obtained, but without specific informed consent from patients. Furthermore, not only were all data securely protected (by the delinking of identifying information from the main data sets) and made available only to the investigators, but they were also analyzed anonymously. Finally, all primary data were collected according to procedures outlined in epidemiology guidelines to strengthen the reporting of observational studies.

\section{Patients}

In total, 222 patients were referred for the management of intentional paraquat ingestion between 2000 and 2012.

\section{Diagnosis of paraquat poisoning}

A presumptive diagnosis of paraquat poisoning was based on exposure history, clinical effects, and physical and laboratory examinations, especially the urine sodium dithionite screening test. The urine sodium dithionite reaction depends on the reduction of paraquat by sodium dithionite under alkaline conditions to form stable, bluecolored radical ions [41]. The generation of a strong navy or dark blue color generally indicates significant paraquat ingestion and often forebodes a poor prognosis. The urine test was used as a paraquat screen, while a confirmatory diagnosis of paraquat poisoning was only possible through the analysis of the blood paraquat concentration (spectrophotometry, Hitachi, Tokyo, Japan). At our hospital, the result of a urine paraquat test is available in 30 minutes, but the result of a blood paraquat test is not available for at least 4 hours.

\section{Definition of AKI}

The definition of AKI was based on the Acute Kidney Injury Network (AKIN) score [42]. 


\section{Definition of acute lung injury and acute respiratory distress syndrome (ARDS)}

Acute lung injury is a continuum of clinical and radiographic changes affecting the lungs, characterized by acute-onset severe hypoxemia, unrelated to left atrial hypertension, occurring at any age. At the severe end of this spectrum lies ARDS. ARDS was defined according to the American-European consensus conference as an acute onset of bilateral pulmonary infiltrates, a ratio of partial pressure of arterial oxygen $\left(\mathrm{PaO}_{2}\right)$ to fraction of inspired oxygen $\leq 200 \mathrm{mmHg}$, and a pulmonary artery occlusion pressure $\leq 18 \mathrm{mmHg}$ or absence of left atrial hypertension [43].

\section{Definition of acute hepatitis}

Acute hepatitis was defined if the serum level of alanine aminotransferase (ALT) was $>72 \mathrm{U} / \mathrm{L}$ (normal $0-36 \mathrm{U} / \mathrm{L}$ ), or total bilirubin was $>1.5 \mathrm{mg} / \mathrm{dL}$ (normal $0-1.3 \mathrm{mg} / \mathrm{dL})$ [2].

\section{Inclusion and exclusion criteria}

Patients were included in this study if they were $>18$ years of age and had urine paraquat tests that displayed dark or navy blue coloring ( $>5 \mathrm{ppm}$ ). Patients were excluded from the study if the paraquat exposure was dermal or intravascular $[44,45]$. Patients were also excluded if paraquat was not detectable in their urine and blood or if they had major comorbidities, such as cancer or heart, lung, renal, or liver diseases. Diagnoses of major comorbidities were based on detailed clinical, physical, and laboratory examinations. Patients with preexisting serum creatinine concentrations $>1.2 \mathrm{mg} / \mathrm{dL}$, ALT concentrations $>36 \mathrm{mg} / \mathrm{dL}$, or total bilirubin concentrations $>3 \mathrm{mg} / \mathrm{dL}$ were also excluded.

\section{Sequential organ failure assessment (SOFA) and AKIN scores}

The following data were collected: baseline demographics, SOFA and AKIN scores 48 hours after admission $\left(\mathrm{SOFA}_{48 \mathrm{~h}}\right.$ and $\mathrm{AKIN}_{48 \mathrm{~h}}$ ), and the time to ARDS (ARDS patients) or nadir $\mathrm{PaO}_{2}$ (non-ARDS patients). The SOFA score consists of six variables, each representing an organ system. Each organ system is assigned a point value from 0 (normal) to 4 (high degree of dysfunction/failure) [14]. The AKIN criteria classify AKI into three stages of severity (stages 1, 2, and 3) [14].

\section{Severity index of paraquat poisoning (SIPP) score}

The SIPP score is calculated as the time to treatment (hours) x serum paraquat concentration (ppm) [24].

\section{Protocol for paraquat detoxification}

The paraquat detoxification protocol $[4,5]$ included gastric lavage with a large amount of $0.9 \%$ saline, followed by $1 \mathrm{~g} / \mathrm{kg}$ activated charcoal and $250 \mathrm{~mL}$ magnesium citrate through a nasogastric tube. Charcoal hemoperfusion with a charcoal-containing (Adsorba, Gambro, Germany) dialysis machine (Surdial, Nipro, Japan) was initiated if the urine paraquat concentration was $>5 \mathrm{ppm}$ [5]. A second session of hemoperfusion was arranged if the urine paraquat concentration was $>5 \mathrm{ppm} 4$ hours after the first hemoperfusion. The protocol also included pulse therapies of cyclophosphamide $(15 \mathrm{mg} / \mathrm{kg} /$ day $)$ for two days and methylprednisolone (1 g/day) for three days [4]. Intravenous dexamethasone ( $20 \mathrm{mg} /$ day) was administered for another 11 days after methylprednisolone pulse therapy. Since cyclophosphamide and methylprednisolone were potentially removable by charcoal hemoperfusion, both agents were administered after the extracorporeal treatment. Pulse therapies with cyclophosphamide and methylprednisolone were repeated if the $\mathrm{PaO}_{2}$ was $<60 \mathrm{mmHg}$ and the duration was more than two weeks after the initial treatment, unless the patient had leucopenia (white cell count $<3000 / \mathrm{m}^{3}$ ). Finally, all patients received normal inspired oxygen therapy $\left(\mathrm{FiO}_{2} 21 \%\right)$ throughout their hospitalization. The rationale for normal inspired oxygen therapy was that an increased $\mathrm{FiO}_{2}$ could increase oxidative stress, and the production of free radicals and superoxide might worsen the acute lung injury and systemic toxicity [46].

\section{Statistical analysis}

Data are expressed as the mean \pm standard deviation or number (percentage), unless otherwise stated. All variables were tested for normal distribution by the Kolmogorov-Smirnov test. Student's $t$ test was used to compare the means of continuous variables and normally distributed data. The Mann-Whitney $U$ test was used for non-normally distributed data. Categorical data were analyzed with the chi-square test. Finally, risk factors were assessed by univariate logistic regression analysis, and variables that were statistically significant $(\mathrm{P}<0.05)$ were included in a multivariate analysis; multiple logistic regression was applied based on forward elimination of data. Calibration was assessed with the HosmerLemeshow goodness-of-fit test to compare the number of observed and predicted AKI and HD cases in risk groups for the entire range of AKI and HD probabilities. Discrimination was assessed by analysis of the area under the receiver operating characteristic curve (AUROC). The AUROCs were compared by a non-parametric approach. AUROC analyses were also used to calculate cutoff values, sensitivities, specificities, and overall correctness. Finally, the cutoff points were calculated in accordance with the best Youden index (sensitivity + specificity -1$)$. 
All statistical tests were two-tailed, with $P$ values $<0.05$ being considered statistically significant. Data were analyzed with SPSS 12.0 software for Windows (SPSS, Inc., Chicago, IL, USA).

\section{Authors' contributions}

CHW and HHC: data collection and manuscript writing; CCH, WHH, SHL, CWH, JFF, WRL, IKW: data analysis; THY: study design and supervision.

\section{ACKNOWLEDGMENTS}

THY is funded by research grants from the Ministry of Science and Technology, Taiwan (MST105-2221-E182A-003) and Chang Gung Memorial Hospital, Taiwan (CLRPG3D0014, CMRPG3F0602, CMRPG3F1161). $\mathrm{CHW}$ is funded by research grants from the Chang Gung Memorial Hospital, Taiwan (CMRPG3A1271, CMRPG5D0081, CMRPG5E0051).

\section{CONFLICTS OF INTEREST}

The authors declare no conflicts of financial interest.

\section{REFERENCES}

1. Lin C, Yen TH, Juang YY, Lin JL, Lee SH. Psychiatric comorbidity and its impact on mortality in patients who attempted suicide by paraquat poisoning during 2000 2010. PLoS One. 2014; 9:e112160. doi: 10.1371/journal. pone. 0112160 .

2. Yang CJ, Lin JL, Lin-Tan DT, Weng CH, Hsu CW, Lee SY, Lee SH, Chang CM, Lin WR, Yen TH. Spectrum of toxic hepatitis following intentional paraquat ingestion: analysis of 187 cases. Liver Int. 2012; 32:1400-6. doi: 10.1111/j.1478-3231.2012.02829.x.

3. Kim SJ, Gil HW, Yang JO, Lee EY, Hong SY. The clinical features of acute kidney injury in patients with acute paraquat intoxication. Nephrol Dial Transplant. 2009; 24:1226-32.

4. Lin JL, Lin-Tan DT, Chen KH, Huang WH, Hsu CW, Hsu HH, Yen TH. Improved survival in severe paraquat poisoning with repeated pulse therapy of cyclophosphamide and steroids. Intensive Care Med. 2011; 37:1006-13. doi: 10.1007/s00134-010-2127-7.

5. Hsu CW, Lin JL, Lin-Tan DT, Chen KH, Yen TH, Wu MS, Lin SC. Early hemoperfusion may improve survival of severely Paraquat-poisoned patients. PLoS One. 2012; 7:e48397. doi: 10.1371/journal.pone.0048397.

6. Lin JL, Liu L, Leu ML. Recovery of respiratory function in survivors with paraquat intoxication. Arch Environ Health. 1995; 50:432-9.

7. Li LR, Sydenham E, Chaudhary B, Beecher D, You C. Glucocorticoid with cyclophosphamide for paraquat-induced lung fibrosis. Cochrane Database Syst Rev. 2014; 8:CD008084. doi: 10.1002/14651858.CD008084.pub4.

8. Molck AM, Friis C. The cytotoxic effect of paraquat to isolated renal proximal tubular segments from rabbits. Toxicology. 1997; 122:123-32.

9. Molck AM, Friis C. Transport of paraquat by isolated renal proximal tubular segments from rabbits. Pharmacol Toxicol. 1998; 83:208-13.

10. Mohamed F, Buckley NA, Jayamanne S, Pickering JW, Peake P, Palangasinghe C, Wijerathna T, Ratnayake I, Shihana F, Endre ZH. Kidney damage biomarkers detect acute kidney injury but only functional markers predict mortality after paraquat ingestion. Toxicol Lett. 2015; 237:140-50. doi: 10.1016/j.toxlet.2015.06.008.

11. Mohamed F, Endre Z, Jayamanne S, Pianta T, Peake P, Palangasinghe C, Chathuranga U, Jayasekera K, Wunnapuk K, Shihana F, Shahmy S, Buckley N. Mechanisms underlying early rapid increases in creatinine in paraquat poisoning. PLoS One. 2015; 10:e0122357. doi: 10.1371/journal.pone.0122357.

12. Kim JH, Gil HW, Yang JO, Lee EY, Hong SY. Serum uric acid level as a marker for mortality and acute kidney injury in patients with acute paraquat intoxication. Nephrol Dial Transplant. 2011; 26:1846-52. doi: 10.1093/ndt/gfq632.

13. Weng CH, Hu CC, Lin JL, Lin-Tan DT, Hsu CW, Yen TH. Predictors of acute respiratory distress syndrome in patients with paraquat intoxication. PLoS One. 2013; 8:e82695. doi: 10.1371/journal.pone.0082695.

14. Weng CH, Hu CC, Lin JL, Lin-Tan DT, Huang WH, Hsu CW, Yen TH. Sequential organ failure assessment score can predict mortality in patients with paraquat intoxication. PLoS One. 2012; 7:e51743. doi: 10.1371/journal.pone.0051743.

15. Seok S, Kim YH, Gil HW, Song HY, Hong SY. The time between paraquat ingestion and a negative dithionite urine test in an independent risk factor for death and organ failure in acute paraquat intoxication. J Korean Med Sci. 2012; 27:993-8. doi: 10.3346/jkms.2012.27.9.993.

16. Moon JM, Chun BJ. The efficacy of high doses of vitamin $\mathrm{C}$ in patients with paraquat poisoning. Hum Exp Toxicol. 2011; 30:844-50. doi: 10.1177/0960327110385633.

17. Gil HW, Yang JO, Lee EY, Hong SY. Clinical implication of urinary neutrophil gelatinase-associated lipocalin and kidney injury molecule- 1 in patients with acute paraquat intoxication. Clin Toxicol (Phila). 2009; 47:870-5. doi: 10.3109/15563650903306651.

18. Koo JR, Kim JC, Yoon JW, Kim GH, Jeon RW, Kim HJ, Chae DW, Noh JW. Failure of continuous venovenous hemofiltration to prevent death in paraquat poisoning. Am J Kidney Dis. 2002; 39:55-9. doi: 10.1053/ajkd.2002.29880.

19. Suntres ZE. Role of antioxidants in paraquat toxicity. Toxicology. 2002; 180:65-77.

20. DeGray JA, Rao DN, Mason RP. Reduction of paraquat and related bipyridylium compounds to free radical metabolites by rat hepatocytes. Arch Biochem Biophys. 1991; 289:145-52. 
21. Konstantinova SG, Russanov EM. Studies on paraquatinduced oxidative stress in rat liver. Acta Physiol Pharmacol Bulg. 1999; 24:107-11.

22. Altun R, Korkmaz M, Yildirim E, Ocal S, Akbas E, Selcuk H. Terlipressin and albumin for type 1 hepatorenal syndrome: does bacterial infection affect the response? Springerplus. 2015; 4:806. doi: 10.1186/s40064-015-1625-z.

23. Moore JK, Love E, Craig DG, Hayes PC, Simpson KJ. Acute kidney injury in acute liver failure: a review. Expert Rev Gastroenterol Hepatol. 2013; 7:701-12. doi: 10.1586/17474124.2013.837264.

24. Sawada Y, Yamamoto I, Hirokane T, Nagai Y, Satoh Y, Ueyama M. Severity index of paraquat poisoning. Lancet. 1988; 1:1333.

25. Senarathna L, Eddleston M, Wilks MF, Woollen BH, Tomenson JA, Roberts DM, Buckley NA. Prediction of outcome after paraquat poisoning by measurement of the plasma paraquat concentration. QJM. 2009; 102:251-9. doi: 10.1093/qjmed/hcp006.

26. Yamamoto I, Saito T, Harunari N, Sato Y, Kato H, Nakagawa Y, Inokuchi S, Sawada Y, Makuuchi H. Correlating the severity of paraquat poisoning with specific hemodynamic and oxygen metabolism variables. Crit Care Med. 2000; 28:1877-83.

27. Lin JL, Wei MC, Liu YC. Pulse therapy with cyclophosphamide and methylprednisolone in patients with moderate to severe paraquat poisoning: a preliminary report. Thorax. 1996; 51:661-3.

28. Lin JL, Leu ML, Liu YC, Chen GH. A prospective clinical trial of pulse therapy with glucocorticoid and cyclophosphamide in moderate to severe paraquat-poisoned patients. Am J Respir Crit Care Med. 1999; 159:357-60.

29. Lin JL, Lin-Tan DT, Chen KH, Huang WH. Repeated pulse of methylprednisolone and cyclophosphamide with continuous dexamethasone therapy for patients with severe paraquat poisoning. Crit Care Med. 2006; 34:368-73.

30. Yoshida T, Tanaka M, Sotomatsu A, Okamoto K. Effect of methylprednisolone-pulse therapy on superoxide production of neutrophils. Neurol Res. 1999; 21:509-12.

31. Balestracci A, Martin SM, Toledo I, Alvarado C, Wainsztein RE. Laboratory predictors of acute dialysis in hemolytic uremic syndrome. Pediatr Int. 2014; 56:234-9. doi: 10.1111/ped.12245.

32. Kielstein JT, Tolk S, Hafer C, Heiden A, Wiesner O, Kuhn C, Hadem J, Hoeper MM, Fischer S. Effect of acute kidney injury requiring extended dialysis on 28 day and 1 year survival of patients undergoing interventional lung assist membrane ventilator treatment. BMC Nephrol. 2011; 12:15. doi: 10.1186/1471-2369-12-15.

33. Lex DJ, Toth R, Cserep Z, Alexander SI, Breuer T, Sapi E, Szatmari A, Szekely E, Gal J, Szekely A. A comparison of the systems for the identification of postoperative acute kidney injury in pediatric cardiac patients. Ann Thorac Surg. 2014; 97:202-10. doi: 10.1016/j.athoracsur.2013.09.014.

34. Geri G, Guillemet L, Dumas F, Charpentier J, Antona M, Lemiale V, Bougouin W, Lamhaut L, Mira JP, Vinsonneau C,
Cariou A. Acute kidney injury after out-of-hospital cardiac arrest: risk factors and prognosis in a large cohort. Intensive Care Med. 2015; 41:1273-80. doi: 10.1007/s00134-015-3848-4.

35. Medeiros P, Nga HS, Menezes P, Bridi R, Balbi A, Ponce D. Acute kidney injury in septic patients admitted to emergency clinical room: risk factors and outcome. Clin Exp Nephrol. 2015; 19:859-66. doi: 10.1007/s10157-014-1076-9.

36. Darmon M, Clec'h C, Adrie C, Argaud L, Allaouchiche B, Azoulay E, Bouadma L, Garrouste-Orgeas M, Haouache H, Schwebel C, Goldgran-Toledano D, Khallel H, Dumenil AS, et al. Acute respiratory distress syndrome and risk of AKI among critically ill patients. Clin J Am Soc Nephrol. 2014; 9:1347-53. doi: 10.2215/CJN.08300813.

37. Seeley EJ. Updates in the management of acute lung injury: a focus on the overlap between AKI and ARDS. Adv Chronic Kidney Dis. 2013; 20:14-20. doi: 10.1053/j. ackd.2012.10.001.

38. Yap SC, Lee HT. Acute kidney injury and extrarenal organ dysfunction: new concepts and experimental evidence. Anesthesiology. 2012; 116:1139-48. doi: 10.1097/ ALN.0b013e31824f951b.

39. Macklin CC. Transport of air along sheaths of pulmonic blood vessels from alveoli to mediastinum: clinical implications. Arch Intern Med. 1939; 64:913-926.

40. Zhou CY, Kang X, Li CB, Li XH, Liu Y, Wang Z, Wang L, Wu T, Mohan C, Hu DY, Peng A. Pneumomediastinum predicts early mortality in acute paraquat poisoning. Clin Toxicol (Phila). 2015; 53:551-6. doi: 10.3109/15563650.2015.1046183.

41. Kuan CM, Lin ST, Yen TH, Wang YL, Cheng CM. Paper-based diagnostic devices for clinical paraquat poisoning diagnosis. Biomicrofluidics. 2016; 10: 034118. doi: 10.1063/1.4953257.

42. Mehta RL, Kellum JA, Shah SV, Molitoris BA, Ronco C, Warnock DG, Levin A, Acute Kidney Injury Network. Acute Kidney Injury Network: report of an initiative to improve outcomes in acute kidney injury. Crit Care. 2007; 11:R31. doi: 10.1186/cc5713.

43. Bernard GR, Artigas A, Brigham KL, Carlet J, Falke K, Hudson L, Lamy M, LeGall JR, Morris A, Spragg R. Report of the American-European consensus conference on ARDS: definitions, mechanisms, relevant outcomes and clinical trial coordination. The Consensus Committee. Intensive Care Med. 1994; 20:225-32.

44. Hsu HH, Chang CT, Lin JL. Intravenous paraquat poisoninginduced multiple organ failure and fatality--a report of two cases. J Toxicol Clin Toxicol. 2003; 41:87-90.

45. Lin NC, Lin JL, Lin-Tan DT, Yu CC. Combined initial cyclophosphamide with repeated methylprednisolone pulse therapy for severe paraquat poisoning from dermal exposure. J Toxicol Clin Toxicol. 2003; 41:877-81.

46. Hoet PH, Demedts M, Nemery B. Effects of oxygen pressure and medium volume on the toxicity of paraquat in rat and human type II pneumocytes. Hum Exp Toxicol. 1997; 16:305-10. doi: 10.1177/096032719701600602. 